\title{
FACTORES DE REDUCCIÓN DE FUERZAS SÍSMICAS PARA EL DISEÑO DE ESTRUCTURAS CON SISTEMAS PASIVOS DE DISIPACIÓN DE ENERGÍA
}

\author{
Danny Arroyo Espinoza ${ }^{(l)}$ y Amador Terán Gilmore ${ }^{(l)}$
}

\begin{abstract}
RESUMEN
Los reglamentos actuales de diseño sísmico no contemplan explícitamente el diseño de estructuras con sistemas de disipación pasiva de energía. Uno de los aspectos más importantes para el desarrollo de una metodología para el diseño de este tipo de estructuras es el planteamiento de métodos para estimar sus fuerzas sísmicas de diseño. Con base en el estudio de la respuesta de sistemas de un grado de libertad ante la acción de acelerogramas registrados en México durante eventos sísmicos recientes, se proponen expresiones para estimar el factor de reducción que debe utilizarse para reducir el espectro de diseño elástico de resistencia para establecer fuerzas sísmicas de diseño para sistemas con diferente capacidad de deformación plástica y de disipación de energía viscosa. Se ofrecen expresiones para suelo firme y suelo blando, en donde el valor del factor de reducción se estima en función del periodo de la estructura, de su demanda máxima de ductilidad y de su coeficiente equivalente de amortiguamiento.
\end{abstract}

\section{SUMMARY}

Current seismic design codes do not contemplate explicitly the design of structures having passive energy dissipation systems. One important issue for the development of a design methodology for these structures is the formulation of methods to estimate their design seismic forces. From the study of the response of single degree of freedom systems subjected to accelerograms recorded during recent seismic events in Mexico, expressions to estimate the value of the reduction factor that should be used to reduce the elastic strength design spectra to establish the design seismic forces for systems having different plastic deformation and viscous energy dissipation capacities, are derived. Expressions corresponding to firm and soft soil sites are presented, in which the value of the reduction factor is estimated as a function of the period, maximum ductility demand and equivalent viscous damping coefficient of the structure.

\section{INTRODUCCIÓN}

Desde hace tiempo, el diseño sismorresistente de las estructuras considera que la resistencia lateral que debe suministrársele a una estructura de ocupación estándar, para que exhiba una

Artículo recibido el 22 de febrero de 2001 y aprobado para su publicación el 11 de septiembre de 2001. Se aceptarán comentarios y/o discusiones hasta cinco meses después de su publicación.

(1) Universidad Autónoma Metropolitana. Departamento de Materiales. Av. San Pablo \# 180. Col. Reynosa, 02200 México, DF.aresda@correo.azc.uam.mx tga@correo.azc.uam.mx 
desempeño estructural adecuado cuando se le sujeta a excitaciones sísmicas de alta intensidad, disminuye conforme aumenta su capacidad de deformación plástica. Con base en lo anterior, el diseño sísmico de las estructuras estándar contempla la posibilidad de proporcionarles una capacidad importante de deformación plástica, como una forma de mantener su resistencia lateral de diseño dentro de un intervalo de valores que hagan que su diseño sea asequible desde un punto de vista económico.

Una de las consecuencias de permitir la incursión significativa de las estructuras en su rango de comportamiento plástico, es la aparición de daño estructural de importancia cuando se les sujeta a excitaciones sísmicas de alta intensidad. Por un lado, aunque el diseño sísmico se plantea de manera que el nivel de daño en la estructura no resulte en su colapso, este daño puede igual conducir a situaciones altamente indeseables, tal como a elevados costos de rehabilitación estructural. Por otro lado, si la estructura incurre de manera excesiva en su rango de comportamiento plástico, las deformaciones resultantes pueden llegar a ser excesivas, lo que se reflejaría en un desempeño no estructural deficiente.

Eventos sísmicos recientes, tales como el de Northridge 1994 (Northridge Reconnaissance Team, 1996) en EE.UU. y el de Kobe 1995 (Mitchell, 1996; Bruneau y Yoshimura, 1996) en Japón, han puesto en evidencia algunos de los problemas asociados al uso de sistemas estructurales tradicionales para resistir las acciones sísmicas durante sismos intensos. Como consecuencia de esto, el uso de sistemas estructurales innovadores ha llamado la atención de investigadores e ingenieros prácticos. Dentro de este contexto, una opción atractiva es el suministro de amortiguamiento adicional a las estructuras por medio de sistemas pasivos de disipación de energía. El buen desempeño sísmico de este tipo de sistemas ha sido puesto en manifiesto en investigaciones analíticas y experimentales (Aiken y Kelly, 1990; Aiken, et al., 1993; Hanson, 1993; Tena y Vergara, 1997; Badillo, 2000), así como durante los eventos sísmicos mencionados con anterioridad (Northridge Reconnaissance Team, 1996).

Actualmente, existe una variedad de dispositivos disipadores de energía que han sido implementados en un número importante de estructuras. En muchos casos, el diseño sismorresistente de estas estructuras ha requerido del apoyo de consultores especializados; mientras que en otros, los ingenieros prácticos han recurrido a la aplicación de diferentes enfoques, particularmente el de amortiguamiento viscoso equivalente. Aunque más adelante se discutirán con más detalle sus fundamentos, baste decir por el momento que este enfoque se centra en la caracterización de la capacidad de disipación de energía del sistema disipador por medio de un coeficiente equivalente de amortiguamiento, que corresponde a una fracción del amortiguamiento viscoso crítico; y que existen algunos lineamientos de diseño sísmico que utilizan este concepto, tal como los que se incluyen en el capítulo 9 de las NEHRP Guidelines for the Seismic Rehabilitation of Buildings (FEMA 273, 1999).

A pesar de lo anterior, existe un vacío importante en cuanto a metodologías y reglamentación para el diseño sísmico de sistemas pasivos de disipación de energía. Uno de los aspectos fundamentales que deben resolverse para hacer posible un diseño racional de estos sistemas, es establecer espectros de resistencia que permitan establecer las fuerzas sísmicas en 
estos dispositivos y la estructura que los contiene. Esto lleva, dentro del contexto de la normatividad actual, a la necesidad de plantear factores de reducción para estos sistemas.

\section{DEFINICIÓN DE FACTOR DE REDUCCIÓN}

Las Normas Técnicas Complementarias para Diseño por Sismo (NTS) del Reglamento de Construcciones para el Distrito Federal (Gaceta Oficial del D.F., 1995) especifican en su Sección 4, titulada REDUCCIÓN DE FUERZAS SÍSMICAS, lo siguiente: "Con fines de diseño las fuerzas sísmicas para análisis estático y las obtenidas del análisis dinámico modal empleando los métodos que fijan estas normas se podrán reducir dividiéndolas entre el factor reductivo Q'."

El factor $Q$ ', se estima en función del factor de comportamiento sísmico $Q$, que entre otras cosas, refleja la capacidad de deformación plástica de la estructura sismorresistente. A través de los factores $Q$ y $Q^{\prime}$, las NTS-95 adoptan una filosofía de diseño que contempla la posibilidad de proporcionar una menor resistencia lateral a la estructura conforme mayor es su capacidad de deformación plástica. Las especificaciones de las NTS-95 contemplan la reducción de las fuerzas sísmicas mínimas requeridas para mantener a la estructura en su rango elástico de comportamiento durante el sismo de diseño, por medio del factor $Q$ ', para obtener las fuerzas sísmicas de diseño. Considerando que las fuerzas sísmicas elásticas corresponden a $Q$ de 1 y a un coeficiente equivalente de amortiguamiento o fracción de amortiguamiento crítico $(\xi)$ de $5 \%$, y que las fuerzas reducidas de diseño para un $Q$ mayor que 1 están asociadas a ese mismo valor de $\xi$, el criterio de la NTS-95 puede expresarse como:

$F S(Q>1, \xi=0.05, T)=F S(Q=1, \xi=0.05, T) / Q^{\prime}(T, Q)$

En la ecuación (1), $F S$ denota fuerza sísmica de diseño, y $T$ el periodo fundamental de vibración de la estructura. Note que tanto $F S$ como $Q$ ' son función, además de lo discutido en el párrafo anterior, del valor de $T$. Con base en lo anterior, puede definirse el factor $Q$ ', dentro del contexto de las NTS-95, conforme a lo siguiente:

$Q\left(T_{0}, Q_{0}\right)=F S\left(Q=1, \xi=0.05, T_{0}\right) / F S\left(Q_{0}, \xi=0.05, T_{0}\right)$

donde $T_{o}$ y $Q_{o}$ son los valores específicos de $T$ y $Q$ asociados a la estructura. Es importante mencionar que dentro del contexto de las NTS-95, el factor $Q$ ' no solo contempla la reducción de las fuerzas sísmicas debido al comportamiento plástico de la estructura, sino también que esta calibrado para que, a juicio de quienes han elaborado el reglamento, resulte en resistencias laterales que lleven a las estructuras a un desempeño estructural adecuado. Dado que en el cuerpo de las normas estas consideraciones se hacen de manera no explícita y poco racional, puede considerarse que la ecuación (2) provee una definición razonable del factor $Q$ ' dentro del contexto de las NTS-95.

En el presente artículo el factor de reducción se definirá con base en la ecuación (2). Sin embargo, por un lado dicho factor contemplará explícitamente la capacidad de deformación plástica de la estructura y su nivel de amortiguamiento viscoso; mientras que por el otro no 
considerará la sobrerresistencia de diseño. La capacidad de deformación plástica de la estructura se caracterizará por medio de su ductilidad $(\mu)$, definida como el cociente entre la máxima demanda de desplazamiento lateral durante la excitación sísmica normalizada por el correspondiente desplazamiento de fluencia. El nivel de amortiguamiento de la estructura se caracterizará por medio de su fracción de amortiguamiento crítico $(\xi)$. Con base en lo anterior, el factor de reducción, $R_{\mu \xi}$, se define como:

$R_{\mu \xi}\left(\mu_{0}, \xi_{0}, T_{0}\right)=F S\left(\mu=1, \xi=0.05, T_{0}\right) / F S\left(\mu_{0}, \xi_{0}, T_{0}\right)$

donde $\mu_{o}, \xi_{o}, T_{o}$ son los valores específicos de $\mu, \xi$ y $T$ asociados a la estructura para la cual se determina el valor de $R_{\mu \xi}$.

\section{ALCANCE PARÁMETRICO}

El estudio que aquí se realizó en dos etapas. Dentro de la primera etapa se utilizaron acelerogramas sintéticos, y se estudió el efecto que sobre el factor de reducción tienen la duración de la fase intensa, y el contenido de frecuencias de la excitación sísmica. En la segunda etapa, se consideraron acelerogramas reales registrados en México durante eventos sísmicos recientes para proponer expresiones que estimen dicho factor. Cabe aclarar que con los resultados obtenidos en la primera etapa se determinó que variables se incluirían en el análisis de regresión llevado a cabo en la segunda etapa, y que en ambas etapas se analizó la influencia que en el valor del factor de reducción tienen las siguientes características mecánicas de la estructura sismorresistente: periodo, demanda máxima de ductilidad y coeficiente equivalente de amortiguamiento.

Para la primera etapa se consideró la respuesta de sistemas de un grado de libertad (S1GL) con comportamiento elastoplástico perfecto. El estudio contempló valores de $T$ de 0.1 a 6 segundos; $\mu$ de 1 a 3 ; y $\xi$ de 0.02 a 0.30 . Se utilizaron 4500 acelerogramas sintéticos, agrupados en tres grupos de 1500 acelerogramas con duración de fase intensa $\left(t_{d}\right)$, definida acorde a Trifunac y Brady (1975), de 10, 20 y 30 segundos, respectivamente. Estos acelerogramas fueron generados filtrando ruidos blancos gaussianos con un filtro Kanai-Tajimi (Tajimi, 1960). El uso de este filtro implica la definición de los parámetros $T_{g}$ y $\xi_{g}$, que aunque no poseen un significado físico preciso, están cercanamente relacionados con el periodo dominante del terreno y con el contenido de frecuencias de la excitación, respectivamente (Lai, 1982; Tung, et al., 1992).

La tabla 1 muestra el valor asignado a los parámetros del filtro Kanai-Tajimi para la generación de los acelerogramas sintéticos. Para cada combinación mostrada y para cada duración se generaron 100 acelerogramas. Cabe aclarar que se eliminaron las frecuencias bajas de las muestras por medio del filtro de Hodder (1983), y que dichas muestras se filtraron en el dominio del tiempo de acuerdo a las indicaciones de Tung et al. (1992), de manera de suministrarles una variación razonable en el tiempo de la intensidad sísmica. La figura 1 muestra la media de los espectros elásticos de resistencia para tres de las familias de acelerogramas sintéticos. Se grafica periodo $(T)$ contra coeficiente sísmico $\left(C_{y}\right)$, definido como la fuerza cortante horizontal máxima que actúa en la base de un sistema sismorresistente normalizada por el peso 
reactivo de dicho sistema. La figura 1 a, obtenida a partir de $T_{g}$ de 0.4 seg. y $\xi_{g}$ de 0.35 , corresponde a acelerogramas sintéticos que modelan movimientos de terreno generados en suelo firme. Se observa un pico en un periodo de $0.4 \mathrm{seg}$, que corresponde al periodo dominante de la excitación, y una amplificación máxima de la aceleración del terreno cercana a 2.5 para dicho periodo. La figura 1c, obtenida a partir de $T_{g}$ de $2.0 \mathrm{seg}$. y $\xi_{g}$ de 0.05 , corresponde a acelerogramas sintéticos que modelan movimientos de terreno generados en la zona del lago del D.F. Para la muestra de suelo blando, se observa una amplificación máxima cercana 5 para un periodo dominante de $2.0 \mathrm{seg}$. Las características de los espectros mostrados en las figuras la y 1c comparan muy bien con aquellas observadas en espectros obtenidos a partir de excitaciones sísmicas reales generadas en suelo firme y suelo blando, respectivamente (Arroyo 2001).

\begin{tabular}{|c|c|c|c|c|c|c|c|c|c|}
\hline \multicolumn{10}{|c|}{ Tabla 1. Combinaciones de $\boldsymbol{T}_{\boldsymbol{g}} \mathbf{y} \boldsymbol{\xi}_{\boldsymbol{g}}$ consideradas } \\
\hline $\boldsymbol{T}_{\boldsymbol{g}}$ & $\xi_{\boldsymbol{g}}$ & $\boldsymbol{T}_{\boldsymbol{g}}$ & $\xi_{\boldsymbol{g}}$ & $\boldsymbol{T}_{\boldsymbol{g}}$ & $\xi_{\boldsymbol{g}}$ & $\boldsymbol{T}_{\boldsymbol{g}}$ & $\xi_{\boldsymbol{g}}$ & $\boldsymbol{T}_{\boldsymbol{g}}$ & $\xi_{\boldsymbol{g}}$ \\
\hline 0.4 & 0.20 & 0.7 & 0.20 & 1.0 & 0.20 & 1.5 & 0.05 & 2.0 & 0.05 \\
\hline 0.4 & 0.35 & 0.7 & 0.35 & 1.0 & 0.35 & 1.5 & 0.20 & 2.0 & 0.20 \\
\hline 0.4 & 0.50 & 0.7 & 0.50 & 1.0 & 0.50 & 1.5 & 0.35 & 2.0 & 0.35 \\
\hline
\end{tabular}
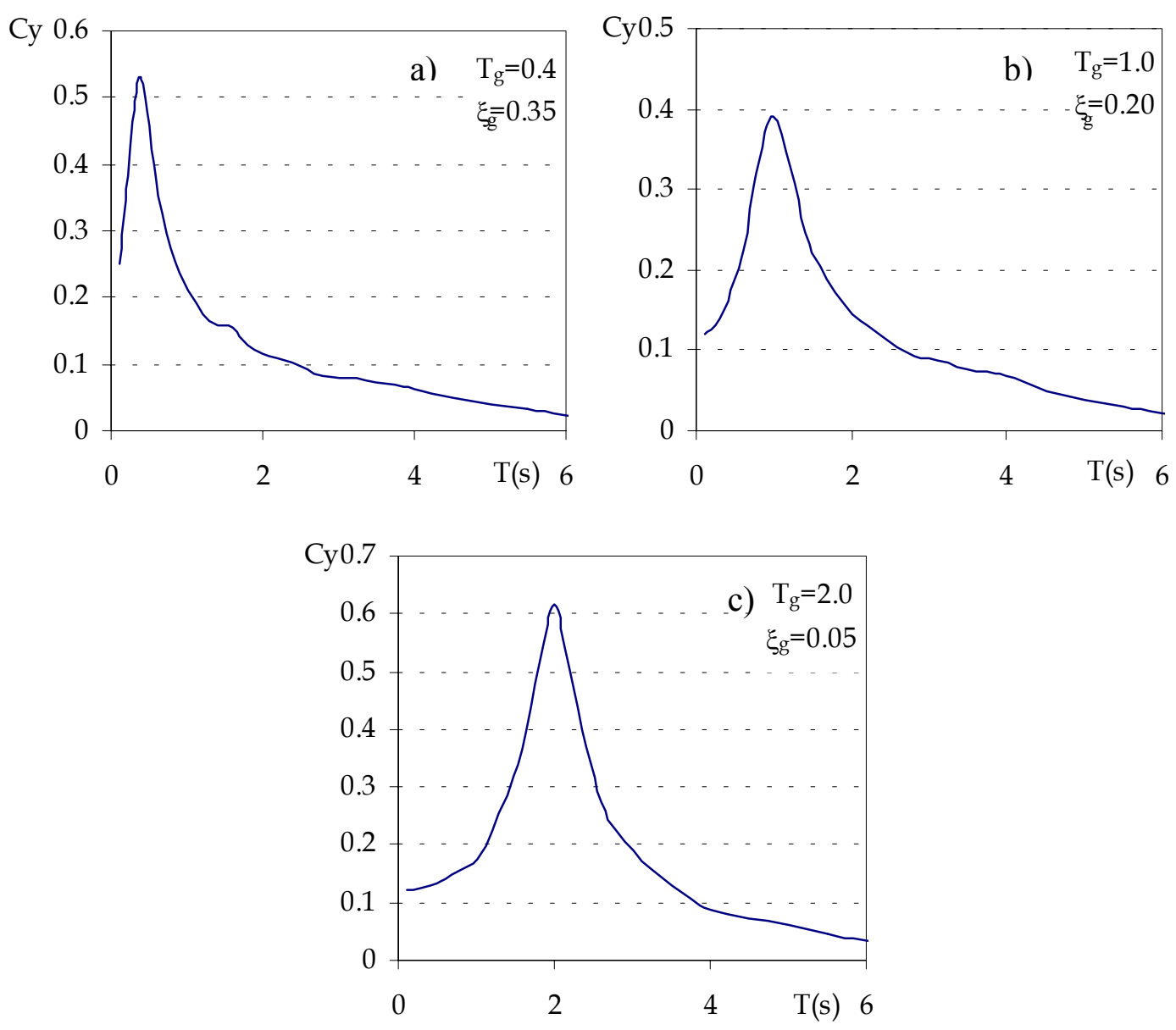

Figura 1. Espectros elásticos promedio de resistencia para acelerogramas sintéticos, $\xi=0.05 \mathrm{y}$ $t_{d}=30 \mathrm{seg}$. 
En la segunda etapa se consideró la respuesta de S1GL con comportamiento elastoplástico perfecto, $\mu$ de 1 a 4 y $\xi$ de 0.02 a 0.30 . En la formulación de una expresión para estimar $R_{\mu \xi}$ para excitaciones características de suelo firme, se consideró un rango de $T$ de 0.1 a 4 segundos. Dado que en el caso de suelos blando la dependencia de $R_{\mu \xi}$ con respecto a $T$ puede expresarse de una forma más conveniente al normalizar $T$ por el periodo dominante de la excitación $\left(T_{g}\right)$, en este caso se consideró un rango de $T / T_{g}$ de 0.1 a 3.2 .

Para el caso de suelo firme, se utilizaron 152 acelerogramas registrados en diferentes sitios de terreno firme a lo largo de la costa del pacífico mexicano y en la zona de lomas del D.F. Cabe mencionar que los acelerogramas utilizados fueron filtrados, para eliminar las frecuencias bajas y corregir problemas de línea base, con el programa Degtra 2000 (Ordaz y Montoya, 2000). Detalles de este filtrado pueden encontrarse en Arroyo (2001). La muestra incluyó acelerogramas con aceleraciones máximas entre $0.005 \mathrm{~g}$ y $0.45 \mathrm{~g}$, y estuvo constituida por una gran cantidad de movimientos menores del terreno, y un número limitado de movimientos de alta intensidad (mas adelante se discutirá en detalle que el valor de $R_{\mu \xi}$ no se ve afectado considerablemente por la intensidad o duración de la excitación sísmica, lo que implica que la muestra utilizada arroja resultados confiables).

Para el caso de suelo blando, se utilizaron 152 acelerogramas registrados en diferentes sitios ubicados en la zona de lago de D.F. Aunque inicialmente el $T_{g}$ de estas excitaciones se definió como el valor de $T$ en que el espectro de velocidad para un $\xi$ de 0.05 alcanza su valor máximo, cabe mencionar que los resultados obtenidos con esta definición no fueron del todo satisfactorios. Como consecuencia, $T_{g}$ se redefinió como el valor de $T$ en que el espectro de energía de entrada para un $\xi$ de 0.05 alcanza su máximo. Los acelerogramas utilizados fueron filtrados para eliminar las frecuencias bajas y corregir problemas de línea base; proceso que se llevó a cabo mediante el filtro de Hodder (1983). Los detalles de este filtrado y una serie de problemas asociados al mismo puede encontrarse en Arroyo (2001). La muestra incluyó acelerogramas con aceleraciones máximas entre $0.01 \mathrm{~g}$ y $0.10 \mathrm{~g}$, y con $T_{g}$ entre 0.8 y 4.7 . Cabe destacar que un gran número de acelerogramas en la muestra estaban caracterizados por $T_{g}$ cercanos a 2. Como en el caso de suelo firme, la muestra estuvo constituida por muchos acelerogramas de baja intensidad, y un número limitado de movimientos con alta intensidad.

Arroyo(2001) presenta información de todos los acelerogramas reales utilizados en el estudio que aquí se reporta, y discute de manera general sus características dinámicas, las cuales se discuten brevemente aquí con ayuda de la figura 2 . La figura 2 presenta, para $\xi$ de 0.05 , la media de los espectros elásticos de resistencia normalizados por la aceleración máxima del terreno, para las muestras de suelo firme y suelo blando. Para el caso de suelo firme, se observa una amplificación máxima promedio cercana a 2.5 y periodo dominante cercano a $0.3 \mathrm{seg}$. Cabe mencionar que este valor de $T_{g}$ coincide con los valores de $T_{g}$ reportados por Tung et al. (1992) para excitaciones generadas en suelo firme. Para la muestra de suelo blando, se observa una amplificación máxima promedio cercana $4.5 \mathrm{y}$, como era de esperarse, un espectro que se maximiza para $T / T_{g}$ de 1.0. Es interesante notar que el espectro promedio para suelo blando incluye un segundo máximo en $T / T_{g}$ cercano a 0.5 , lo que indica que muchos acelerogramas en la muestra exhibieron más de un pico en su espectro de resistencia. 


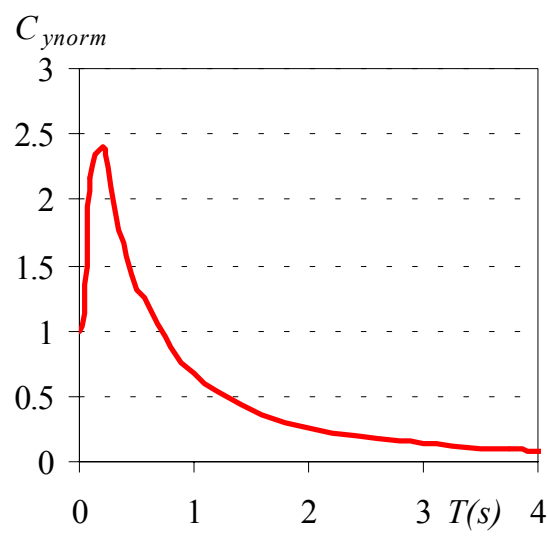

a) Suelo firme

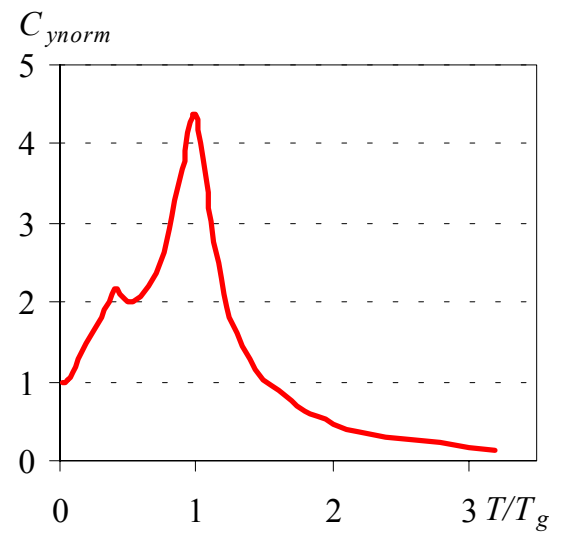

b) Suelo blando

Figura 2. Espectros elásticos promedio de resistencia para acelerogramas reales, $\xi=0.05$

\section{ESTUDIOS PREVIOS Y RESULTADOS OBTENIDOS EN LA PRIMERA ETAPA}

Los resultados obtenidos en la primera etapa, así como una revisión bibliográfica de estudios previos en el tema se utilizaron con dos objetivos: primero, para definir las características de las excitaciones sísmicas y las características mecánicas de las estructuras sismorresistentes que deberían utilizarse durante las regresiones planteadas durante la segunda etapa; y segundo, para definir criterios de selección para los acelerogramas que pasaron a formar parte de las muestras utilizadas en dichas regresiones. Cabe mencionar que aquí solo se presentan las conclusiones derivadas de esta primer etapa, y se comparan con las conclusiones obtenidas por otros investigadores. Una presentación detallada de los resultados obtenidos durante la primer etapa, así como de los trabajos de dichos investigadores puede encontrarse en Arroyo (2001).

En primer lugar, el estudio de la respuesta de S1GL ante excitaciones sintéticas con diferente contenido de frecuencias muestra una clara dependencia de los valores de $R_{\mu \xi}$ con el contenido de frecuencias de la excitación, lo que a su vez implica una dependencia importante con respecto al tipo de suelo donde se genera el movimiento sísmico del terreno. En particular, se observó que para excitaciones características de suelo firme y $\xi$ de 0.05 , el valor $R_{\mu \xi}$ tiende a uno para $T$ pequeño. Para sistemas que desarrollan una ductilidad de $\mu$ durante la excitación sísmica, se observó que el valor de $R_{\mu \xi}$ crece rápidamente conforme el valor de $T$ se incrementa a partir de cero, hasta que alcanza un máximo con valor ligeramente mayor que $\mu$ para $T$ cercano a $T_{g}$, tras lo cual exhibe una ligera reducción hasta alcanzar el valor de $\mu$ para $T$ grande.

En el caso de suelo blando y $\xi$ de 0.05 , se observó que $R_{\mu \xi}$ también tiende a uno para $T$ pequeño. Para sistemas que desarrollan una ductilidad de $\mu, R_{\mu \xi}$ crece conforme el valor de $T$ se incrementa a partir de cero, hasta alcanzar un valor significativamente mayor que $\mu$ para $T$ cercano a $T_{g}$, tras lo cual exhibe una importante reducción hasta alcanzar valores menores que $\mu$ para $T$ grande. 
Dado que el uso de acelerogramas sintéticos arrojó tendencias para $R_{\mu \xi}$ muy similares a las observadas por varios investigadores, entre ellos Nassar y Krawinkler (1991), Miranda (1993) y Ordaz y Pérez (1998), se consideró que el uso de acelerogramas sintéticos es una herramienta razonable para estudiar en detalle el efecto que en valor de $R_{\mu \xi}$ tienen algunas características, tanto de la excitación sísmica como de la estructura, que no fueron consideradas en estudios previos.

Después de comparar los resultados obtenidos a partir de acelerogramas sintéticos con duración de fase intensa definida acorde a Trifunac y Brady (1975) de 10, 20 y 30 segundos, se concluyó que dicha duración tiene poca influencia en $R_{\mu \xi}$. Aunque el efecto de la duración no ha sido considerado directamente por otros autores, cabe mencionar que la poca influencia de la duración en el valor de $R_{\mu \xi}$ es consistente con lo planteado por Miranda (1993) y Nassar y Krawinkler (1991), quienes han observado que el valor de $R_{\mu \xi}$ no se ve afectado de manera importante por la intensidad de la excitación sísmica o por su distancia epicentral. A partir de esto se tomaron dos decisiones: primero, excluir del análisis de regresión la duración de la excitación sísmica; y segundo, plantear para la regresión muestras que tuvieran sismos reales con diferente intensidad y registrados a muy diferentes distancias del epicentro del evento sísmico.

A partir de comparar los resultados obtenidos de acelerogramas sintéticos con diferente contenido de frecuencia (recuerde que este contenido se define a partir de $T_{g}$ y $\xi_{g}$ ), se concluyó que este tiene una influencia importante en el valor de $R_{\mu \xi}$. Para que el lector pueda poner en contexto las afirmaciones que se hacen a continuación, debe considerar que, como lo sugiere la tabla 1, un incremento en el periodo dominante de la excitación suele verse reflejado en una reducción en la banda de frecuencias dominantes alrededor de este periodo (esto es, un incremento de $T_{g}$ suele verse acompañado con un decremento de $\xi_{g}$ ). Dentro de las tendencias observadas destacan las siguientes: primero, un incremento en el periodo dominante de la excitación sísmica se ve reflejado en un aumento significativo del valor máximo de $R_{\mu \xi}$, el cuál se da para $T$ cercano a $T_{g}$; segundo, para valores pequeños de $T_{g}$ (por ejemplo 0.4 segundos, valor característico de suelos firmes), una reducción en el contenido de frecuencias no tiene influencia importante en el valor de $R_{\mu \xi}$ (note en la tabla 1 que el valor de $\xi_{g}$ osciló entre 0.20 y 0.50 para $T_{g}$ de 0.4 segundos); y tercero, conforme el valor de $T_{g}$ se incrementa, la reducción en el contenido de frecuencias tiene mayor influencia en el valor de $R_{\mu \xi}$, hasta llegar a tener una influencia de consideración para valores grandes de $T_{g}$ (por ejemplo 2.0 segundos, valor característico de suelos blandos, para el cual se observó, entre otras cosas, un incremento considerable en el valor máximo de $R_{\mu \xi}$ cuando el valor de $\xi_{g}$ pasa de 0.20 a 0.05 ). A partir de lo anterior se decidió conformar dos muestras de acelerogramas, una correspondiente a suelo firme y la otra para suelo blando, y llevar a cabo análisis de regresión para cada una de ellas. Además, y dado que la muestra correspondiente a suelo blando incluyó acelerogramas con muy diferente $T_{g}$ y por tanto contenidos de frecuencia, se consideró importante hacer submuestras para suelo blando; con el objetivo de estudiar lo robusto de dicha muestra.

Finalmente se estudió la interacción entre $\xi$ y $\mu$, y su influencia en los valores de $R_{\mu \xi}$. Se observó que aún para la mayor $\mu$ considerada en el estudio (con valor de 4), un incremento en el valor de $\xi$ se refleja en un incremento importante en el valor de $R_{\mu \xi}$; mientras que aún para el 
mayor $\xi$ considerado en el estudio (con valor de 0.30 ), un incremento en el valor de $\mu$ se refleja en un incremento importante en el valor de $R_{\mu \xi}$. Lo anterior implica que la combinación de comportamiento plástico con disipación pasiva de energía representa una alternativa viable para resistir las acciones sísmicas y, por tanto, que es importante plantear expresiones para estimar el valor de $R_{\mu \xi}$ para estructuras con diferentes combinaciones de $\mu$ y $\xi$.

\section{SEGUNDA ETAPA: REGRESIÓN}

Una vez conformadas las muestras de acelerogramas reales, se procedió a hacer un análisis de regresión para plantear expresiones que, en función de las variables identificadas como importantes durante la primera etapa del estudio, sean capaces de estimar confiablemente el valor de $R_{\mu \xi}$.

\section{Suelo firme}

Después de algunas propuestas preliminares, se consideró estimar el valor de $R_{\mu \xi}$ para suelos firmes a partir de una familia de curvas con la siguiente forma.

$$
R_{\mu \xi}=\left[\phi\left(\frac{T^{\alpha}}{\beta+T^{\alpha}}\right)+1\right]^{\theta}
$$

La forma básica de la ecuación (4) esta definida por el término $T^{\alpha} /\left(\beta+T^{\alpha}\right)$, el cuál tiende a cero conforme $T$ se aproxima a cero, y tiende a uno conforme el valor de $T$ se incrementa. El valor máximo de $R_{\mu \xi}$ depende de $\phi$ y $\theta$, mientras que el valor de $\theta$ define la velocidad de caída del valor de $R_{\mu \xi}$ a partir de que alcanza ese máximo. Mediante un análisis de regresión basado en mínimos cuadrados para reducir el error cuadrático máximo, se encontraron las siguientes expresiones para $\alpha, \beta, \phi$ y $\theta$.

$$
\begin{aligned}
& \beta=0.16 \\
& \alpha=1.2 \\
& \phi=\mu(3.4 \xi+1.4)-1.2 \xi^{-0.1} \\
& \theta=\frac{0.93-\frac{(\mu-4)}{67}}{T^{\frac{1}{4 \mu}}}
\end{aligned}
$$

En la figura 3 se comparan, para $\xi$ de $0.05,0.10,0.20$ y 0.30 , y $\mu$ de $1,1.5,2,3$ y 4 , los factores de reducción promedio reales (líneas continuas) para la muestra de suelo firme con los valores estimados con la ecuación (4) (líneas discontinuas). Puede observarse una excelente coincidencia entre los valores arrojados por las expresiones propuestas y los valores reales de $R_{\mu \xi}$. 
Es importante mencionar que la aplicación de la ecuación (4) puede ser incómoda para algunos ingenieros de la práctica, particularmente en cuanto a la estimación del exponente $\theta$ se refiere. Para proponer expresiones prácticas para estimar $R_{\mu \xi}$, debe considerarse un balance entre la precisión requerida durante el diseño sísmico y lo complejo de la expresión matemática propuesta. Para ilustrar este punto, considere una simplificación de la ecuación (4) conforme lo siguiente:

$$
R_{\mu \xi}=\phi\left(\frac{T^{\alpha}}{\beta+T^{\alpha}}\right)+1
$$

en la cual:

$\beta=0.002$

$\alpha=1.2$

$\phi=\mu(2.2 \xi+1)-0.75 \xi^{-0.1}$
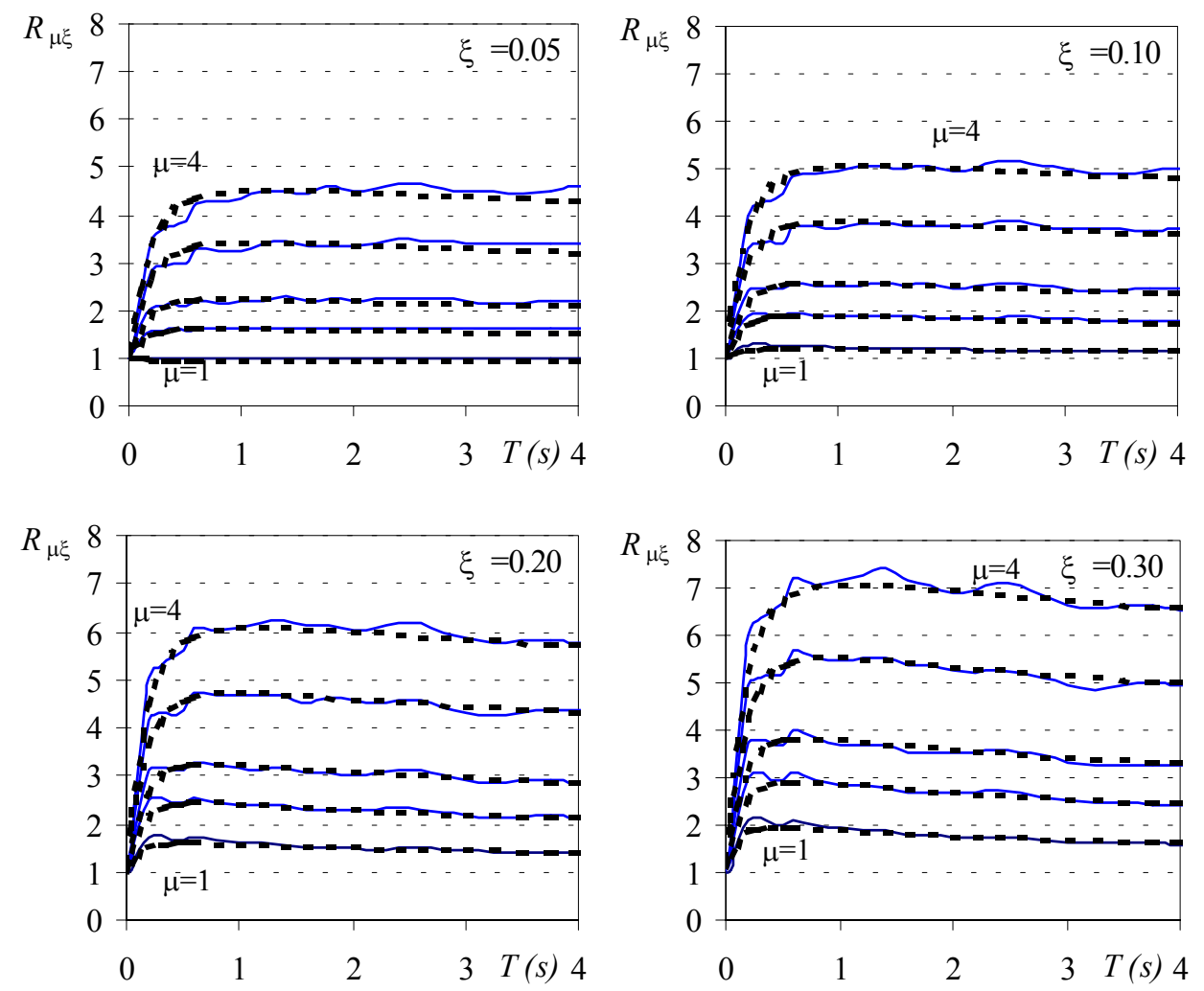

Figura 3. Comparación de factor de reducción estimado (ecuación 4) y real para suelo firme

En la figura 4 se comparan los valores de $R_{\mu \xi}$ obtenidos con la ecuación (9) (líneas discontinuas) con los factores de reducción promedio reales (líneas continuas). Puede observarse que la simplificación de la ecuación (4) para obtener la ecuación (9) resulta en que la predicción del valor de $R_{\mu \xi}$ se aleje un poco del valor real, particularmente para la combinación de $\xi$ alto y $\mu$ 
baja. Sin embargo, puede considerarse que, dentro de un contexto práctico y para el rango de $T$ considerado en este estudio, la ecuación (9) conduce a valores razonables de $R_{\mu \xi}$.
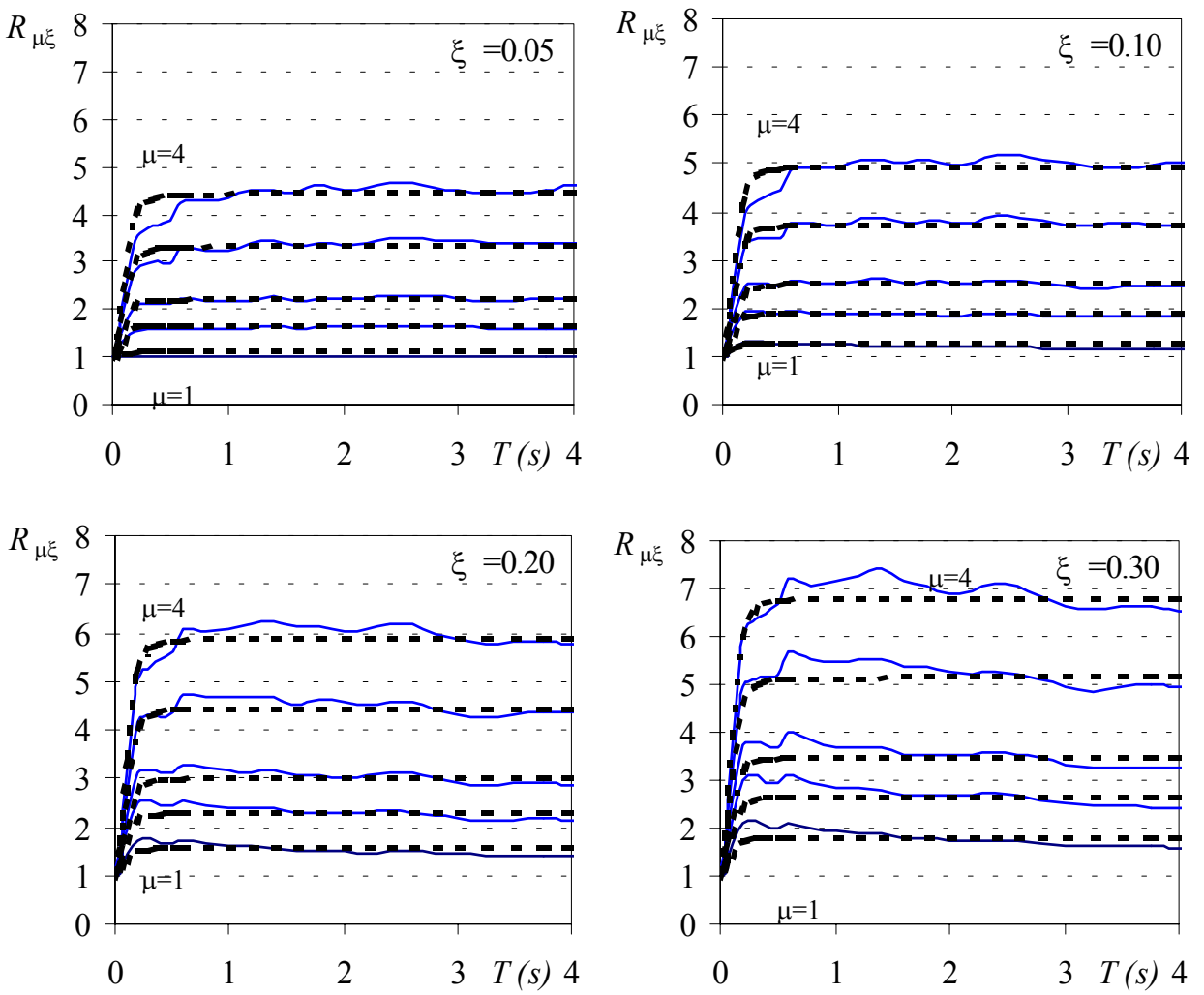

Figura 4. Comparación de factor de reducción estimado (ecuación 9) y real para suelo firme

\section{Suelo blando}

Después de algunas propuestas preliminares, se consideró estimar el valor de $R_{\mu \xi}$ para suelos blandos a partir de una familia de curvas con la siguiente forma.

$R_{\mu \xi}=\frac{\left(\frac{T}{T_{g}}\right)^{\theta}}{b+\left|\frac{T}{T_{g}}-1\right|} \varphi+1$

La forma básica de la ecuación (13), $\left(T / T_{g}\right) /\left(b+\left|T / T_{g}-1\right|\right)$, se planteó de manera que fuera muy similar a la forma básica planteada para suelo firme. La forma básica para suelo blando tiende a cero conforme el valor de $T$ tiende a cero, y adquiere valores considerablemente mayores a los estimados por la forma básica para suelo firme para valores de $T$ cercanos a $T_{g}$. El valor máximo de $R_{\mu \xi}$ para suelos blandos depende de los valores de $b, \varphi$ y $\theta$; mientras que el valor de $\theta$ define la velocidad de caída de $R_{\mu \xi}$ a partir de ese máximo. Mediante un análisis de regresión 
basado en mínimos cuadrados para reducir el error cuadrático máximo, se encontraron las siguientes expresiones para $b, \varphi$ y $\theta$.

$b=0.175$

$\varphi=0.92 \mu \xi^{0.17}+\xi-0.6$

$\theta=\frac{\mu^{4}}{2.5+\mu^{4}}$

En la figura 5 se comparan, para $\xi$ de $0.05,0.10,0.20$ y 0.30, y $\mu$ de $1,1.5,2,3$ y 4 , los factores de reducción promedio reales (líneas continuas) para la muestra de suelo blando con los valores estimados con la ecuación (13) (líneas discontinuas). Puede observarse una buena coincidencia entre estos valores. Cabe mencionar que los valores reales de $R_{\mu \xi}$ correspondientes al primer pico identificado en el espectro elástico de resistencia (ver figura 2 b) son mayores que los obtenidos con la ecuación (13); sin embargo, corregir esta deficiencia resultaría en una expresión mucho mas compleja que la propuesta. Una vez más se enfatiza, ahora para suelo blando, la necesidad de equilibrar la precisión requerida durante el diseño sísmico con la complejidad de la expresión propuesta para estimar $R_{\mu \xi}$.
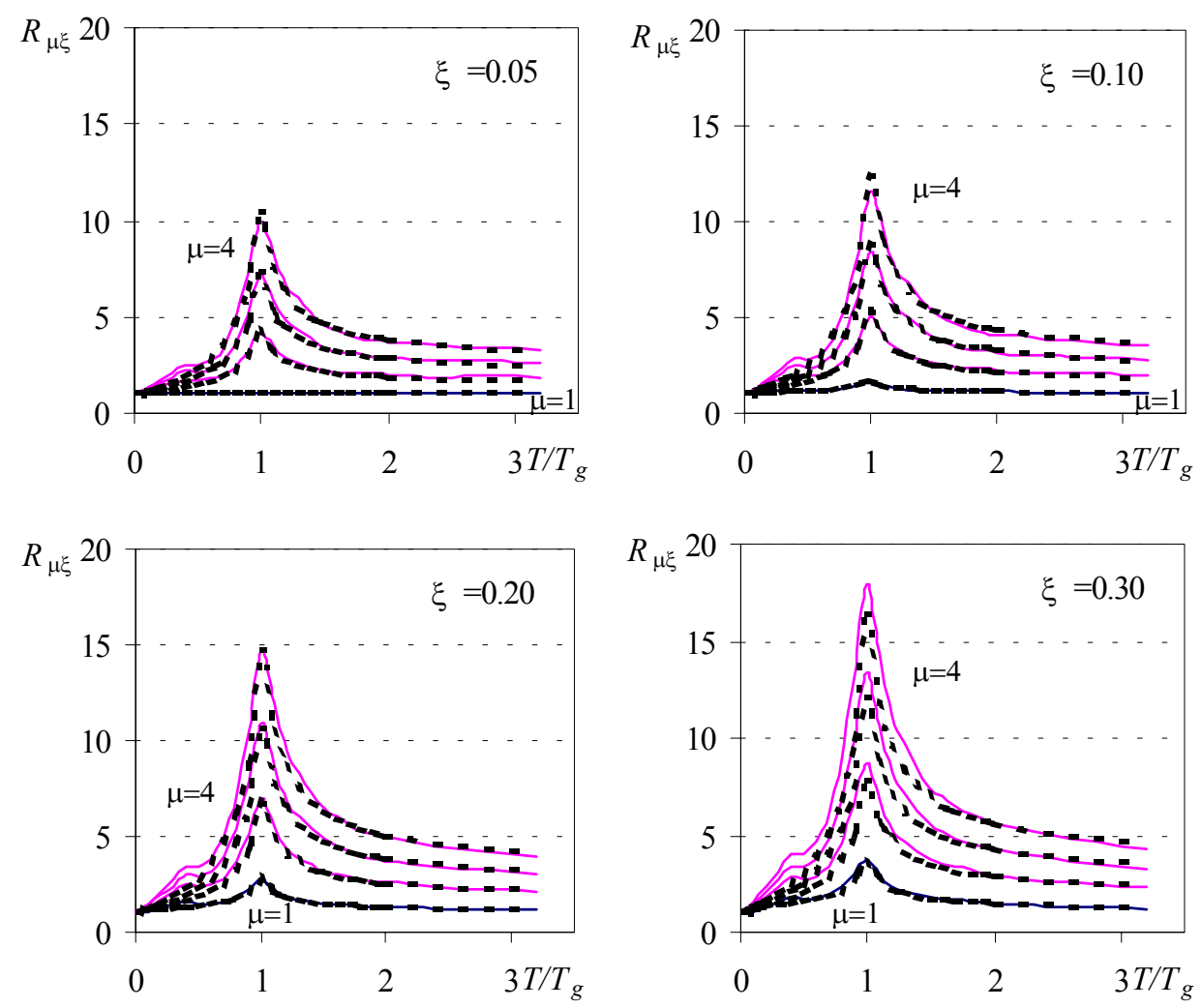

Figura 5. Comparación de factor de reducción estimado y real para suelo blando 


\section{OBSERVACIONES}

\section{Condiciones de frontera}

Las ecuaciones (4) y (13) permiten estimar $R_{\mu \xi}$ para sistemas ubicados en sitios de suelos firme y blando, respectivamente, en función de su periodo, su capacidad máxima de deformación plástica y su capacidad de disipación de energía viscosa. Para el caso de suelo blando, también se considera el periodo predominante de la excitación.

Las ecuaciones (4) y (13) deben satisfacer algunas condiciones de frontera que pueden establecerse a partir de algunos fundamentos de la dinámica estructural. En primer lugar, el valor de $R_{\mu \xi}$ debe tender a uno, independientemente de los valores de $\mu$ y $\xi$ y del tipo de suelo, conforme el valor de $T$ tiende a cero. Esto se debe a que la aceleración máxima que experimenta un sistema infinitamente rígido durante una excitación sísmica es igual a la aceleración máxima del terreno. Las ecuaciones propuestas cumplen satisfactoriamente con esta condición. En segundo lugar, el valor de $R_{\mu \xi}$ debe tender a $\mu$, independientemente de los valores de $\mu$ y $\xi$ y del tipo de suelo, conforme el valor de $T$ tiende a infinito. Esto se debe a que el desplazamiento relativo máximo para un sistema muy flexible es prácticamente igual al desplazamiento máximo del terreno. Note que las ecuaciones propuestas no cumplen con esta condición, ya que han sido planteadas para rangos de $T$ cuyo límite superior esta muy lejano de los valores de $T$ para los cuales se cumple la segunda condición.

Ahondando un poco en lo que se refiere a la segunda condición mencionada en el párrafo anterior, cabe mencionar que se observó que el valor de $T$ para el cual el desplazamiento relativo de la estructura (ya sea elástico o inelástico) es igual al desplazamiento máximo del terreno depende, de manera importante, de dos factores: el filtrado del acelerograma y la resistencia del sistema sismorresistente. Al respecto, se encontró que por lo general, este valor de $T$ disminuye conforme se filtre un mayor rango de frecuencias bajas, y conforme la resistencia de los sistemas disminuye con respecto a su resistencia elástica mínima. En particular, se encontró que en el caso de suelo firme, la segunda condición se cumple con rigurosidad a partir de un $T$ entre 15 y 20 segundos, mientras que para suelo blando, dicha condición se satisface para $T / T_{g}$ entre 8 y 12 . Note que ambos límites están muy por arriba de los valores de $T$ considerados en las regresiones.

Una tercera condición que deben cumplir las ecuaciones propuestas, esta última derivada de la definición presentada en la ecuación (3), es que $R_{\mu \xi}$ para $\mu$ de1 y $\xi$ de 0.05 debe ser igual a uno para todo $T$. Para estas condiciones, las expresiones propuestas dan lugar a valores entre $0.96 \mathrm{y}$ 1.00 para suelo firme y entre 1.00 y 1.02 para suelo blando, valores que para fines prácticos pueden considerarse aceptables.

\section{Comparación con otras propuestas}

Hasta el momento, varios investigadores han planteado expresiones para estimar los factores de reducción asociados a las estructuras sismorresistentes (Nassar y Krawinkler, 1991; Miranda, 
1993; Ordaz y Pérez, 1998). Normalmente estos estudios se han concentrado en sistemas de un grado de libertad (S1GL), con comportamiento elastoplástico y $\xi$ de 0.05 , sujetos a excitaciones sísmicas características de suelo firme. Dentro de los estudios hechos para excitaciones sísmicas características de suelo blando destacan los de Miranda (1993) y los de Ordaz y Pérez (1998); estos últimos investigadores han estudiado el efecto del valor de $\xi$ en el valor del factor de reducción. En cuanto a reglamentos de diseño, el FEMA 273 (1999) establece criterios para estimar espectros elásticos de resistencia para sistemas con valores de amortiguamiento equivalente diferente a 0.05 .

Las figuras 6 y 7 comparan, para $\xi$ de 0.05 , los resultados obtenidos con las ecuaciones (4) y (13), respectivamente, con aquellos obtenidos con las ecuaciones propuestas por los investigadores mencionados anteriormente. Cabe mencionar que algunas de las diferencias identificadas pueden deberse a que las diferentes expresiones han sido obtenidas a partir de diferentes muestras de acelerogramas.

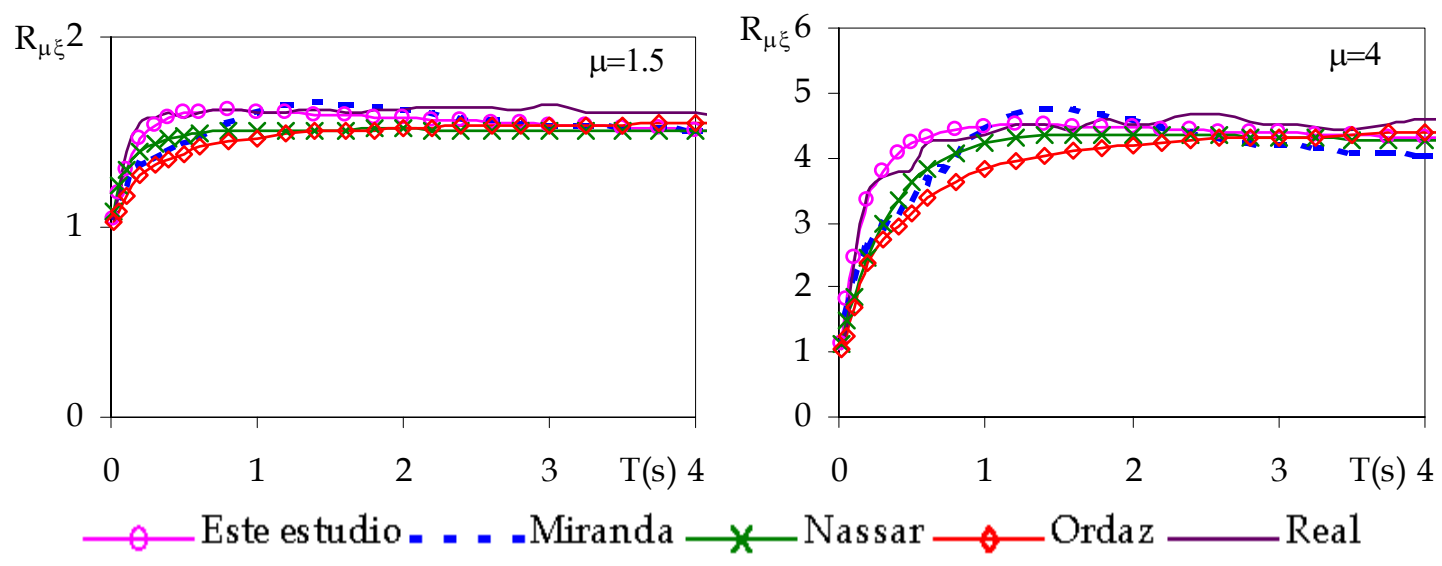

Figura 6. Propuestas para estimar $R_{\mu \xi}$ en suelo firme, $\xi$ de $5 \%$

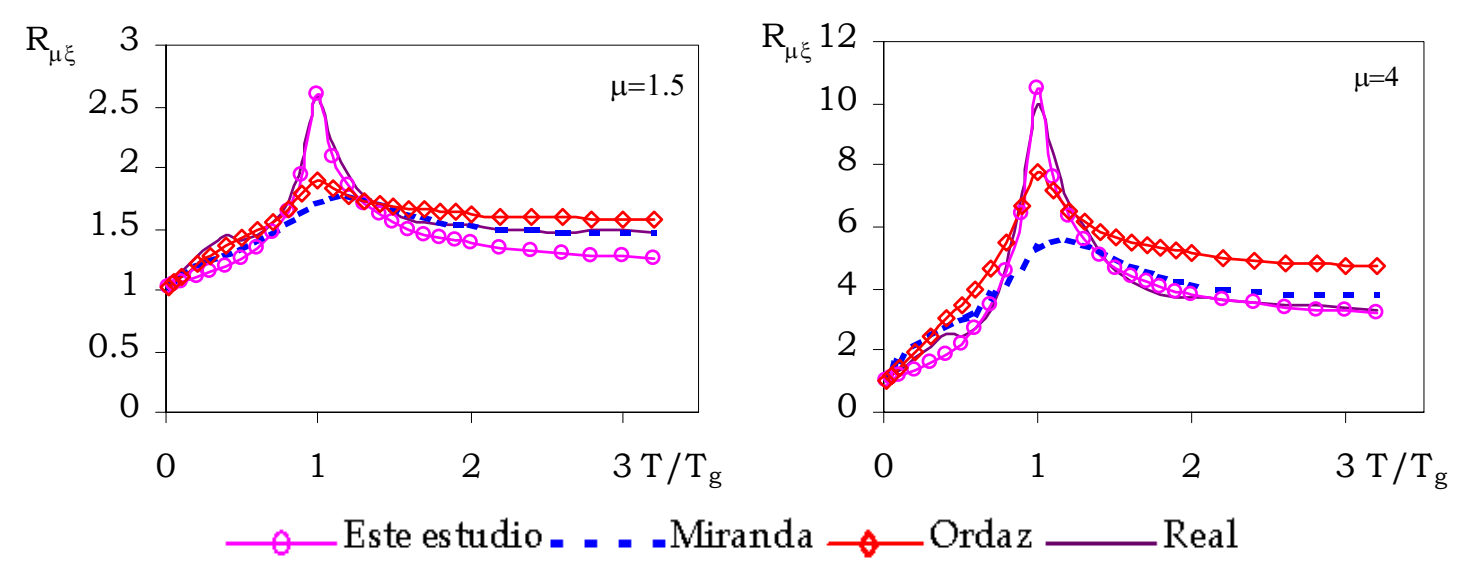

Figura 7. Propuestas para estimar $R_{\mu \xi}$ en suelo blando, $\xi$ de $5 \%$

La figura 6 muestra que todas las expresiones consideradas para suelo firme arrojan resultados muy similares para $\xi$ de 0.05 . Para el caso de suelo blando y $\xi$ de 0.05 , se observa que la ecuación (13) tiende a subestimar ligeramente el valor de $R_{\mu \xi}$ para $\mu$ pequeña combinada con $T$ 
grande; que la expresión propuesta por Ordaz y Pérez (1998) tiende a subestimar ligeramente $R_{\mu \xi}$ para $T$ cercano a $T_{g}$ y a sobreestimarlo para $T$ grande, sobreestimación particularmente importante para $\mu$ grande; y que la expresión propuesta por Miranda (1993) conduce a una subestimación importante de $R_{\mu \xi}$ para $T$ cercano a $T_{g}$. Se aclara que aunque Miranda (1993) ajustó su expresión para suelo blando de manera que tomará en cuenta la incertidumbre en la estimación de $T$ y $T_{g}$, su expresión no parece reflejar adecuadamente la magnitud del pico encontrado en este estudio para suelos blandos.

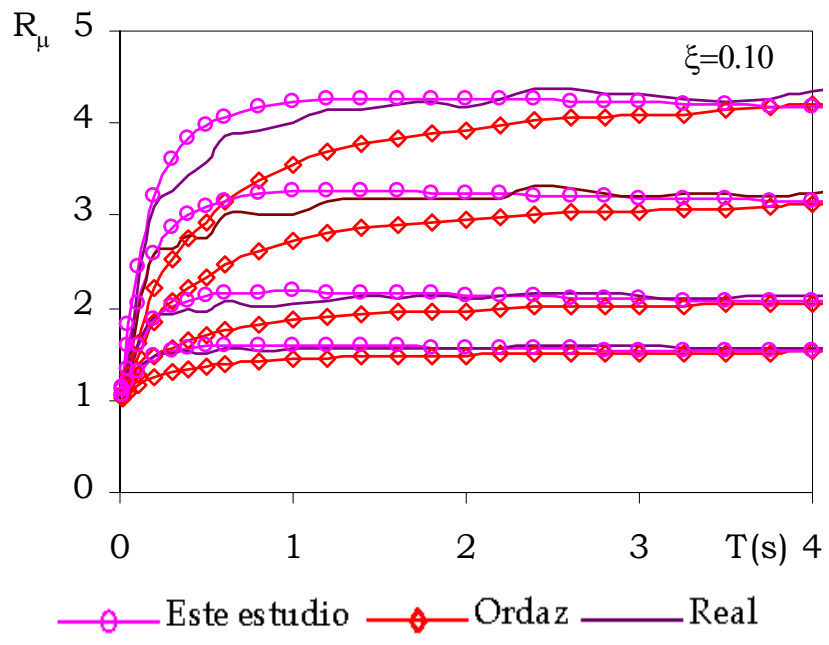

Figura 8. Propuestas para estimar $R_{\mu \xi}$ en suelo firme, $\xi$ de $10 \%$

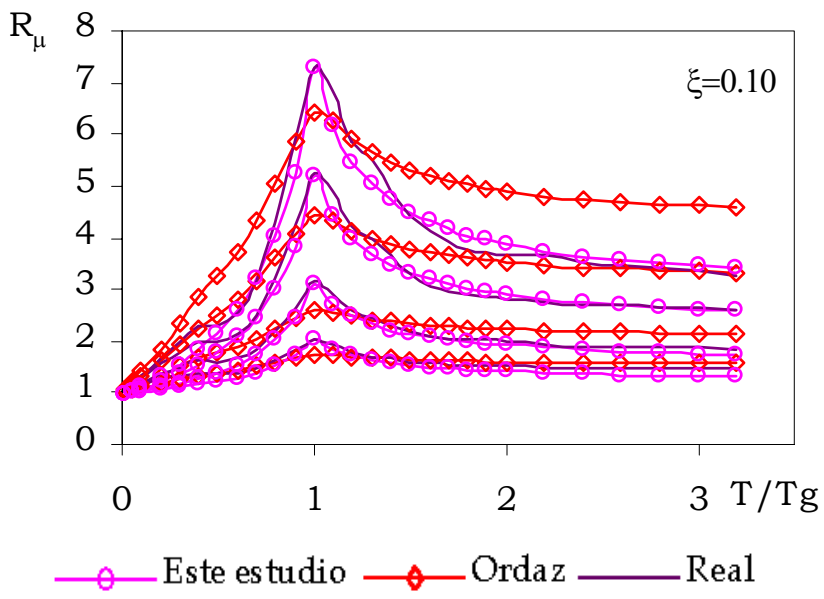

Figura 9. Propuestas para estimar $R_{\mu \xi}$ en suelo blando, $\xi$ de $10 \%$

Las figuras 8 y 9 comparan, para suelo firme y suelo blando, respectivamente, los valores de $R_{\mu \xi}$ obtenidos para $\xi$ de 0.10 a partir de la expresión propuesta por Ordaz y Pérez (1998) y de las ecuaciones (4) y (13). Puede concluirse que las ecuaciones (4) y (13) arrojan resultados mas cercanos a los valores reales de $R_{\mu \xi}$, tanto para suelo firme como para suelo blando. Cabe aclarar que las ecuaciones (4) y (13) se han planteado para estimar, a partir del espectro elástico de resistencia para un $\xi$ de $5 \%$, las fuerzas sísmicas de diseño para sistemas con diferentes 
combinaciones de $\mu$ y $\xi$; mientras que la propuesta de Ordaz y Pérez (1998) requiere estimar los espectros elásticos de resistencia y desplazamiento para el valor de $\xi$ para el cual se desea estimar las fuerzas sísmicas de diseño.

\section{Consideraciones especiales para suelo blando}

Resulta interesante mencionar que los valores de $R_{\mu \xi}$ para suelo blando muestran variaciones importantes en función del valor de $T_{g} \mathrm{y}$ el contenido de frecuencias de los acelerogramas. Para ilustrar esto, la figura 10 compara el $R_{\mu \xi}$ promedio para acelerogramas con $T_{g}$ de 2 segundos y con $T_{g}$ de 4 segundos. En dicha figura se consideró un $\xi$ de 0.05 y $\mu$ de $1.5,2,3$ y 4 . Se aclara que la muestra utilizada para obtener la ecuación (13) incluyó acelerogramas registrados en diferentes sitios ubicados en la zona de terreno blando del D.F., y que incluyó una mayoría de acelerogramas con $T_{g}$ cercano a 2 segundos (Arroyo, 2001). Se observa que aunque los valores máximos de $R_{\mu \xi}$ son muy similares para ambos $T_{g}$ (ligeramente mayores para $T_{g}$ de 4 segundos), la velocidad de caída del valor de $R_{\mu \xi}$ para $T / T_{g}$ mayor que uno es mayor para $T_{g}$ de 4 segundos. Lo anterior refleja que los sitios con $T_{g}$ de 4 segundos poseen un menor contenido de frecuencias alrededor de $T_{g}$ que aquellos con $T_{g}$ de 2 segundos.
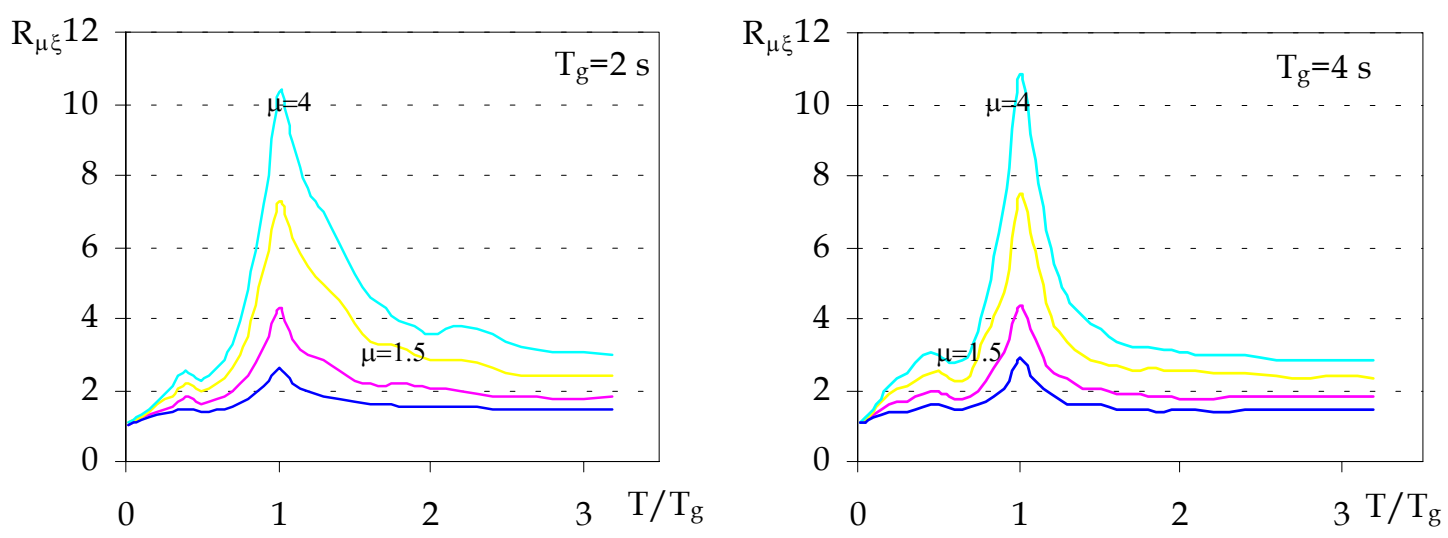

Figura 10. $R_{\mu \xi}$ para sitios de suelo blando con diferente $T_{g}, \xi$ de 0.05

Las diferencias ilustradas en la figura 10 implican que es necesario tener mucho cuidado para conformar muestras de acelerogramas de suelo blando para llevar a cabo un análisis de regresión. En particular, el uso de los valores de $R_{\mu \xi}$ mostrados en la figura 10a para estimar los valores de $R_{\mu \xi}$ para excitaciones con $T_{g}$ de 4 segundos, llevaría a sobreestimar el valor de $R_{\mu \xi}$ para $T / T_{g}$ ligeramente mayor que uno. Una solución a este problema implica analizar cuidadosamente el balance entre la precisión requerida durante el diseño sísmico, el manejo de la incertidumbre involucrada, y la complejidad de la expresión propuesta para estimar $R_{\mu \xi}$. En todo caso y de considerarse necesario, sería posible hacer una consideración explícita de los valores de $T_{g}$ y $\xi_{g}$ en los análisis de regresión. 
A diferencia del caso de suelo firme, donde el valor de $R_{\mu \xi}$ es insensible al valor de $T$ en un amplio rango de valores de $T$, en suelo blando existe una variación muy importante de $R_{\mu \xi}$ con el valor de $T$, particularmente cuando este esta cercano al valor de $T_{g}$. En este rango de valores, una pequeña variación de $T$ se ve reflejado en un cambio considerable de $R_{\mu \xi}$, lo que refleja la importancia de considerar para este caso la incertidumbre involucrada en la determinación de los valores de $T$ y $T_{g}$. Miranda (1993) comenta un criterio simple para contemplar dicha incertidumbre en las expresiones para estimar $R_{\mu \xi}$. Con base en la confiabilidad implícita en los códigos de diseño sísmico y a la magnitud de la incertidumbre involucrada, será necesario modificar las ecuaciones (4) y (13), o en su caso cualquier otra expresión, para su uso práctico.

\section{Aspectos relevantes para el uso y diseño sísmico de sistemas disipadores de energía}

El buen desempeño sísmico observado en estructuras con dispositivos disipadores de energía durante eventos sísmicos recientes, plantea la necesidad de estudiar su posible uso en la República Mexicana. En particular, los estudios hechos hasta la fecha sugieren que estos dispositivos podrían ser una alternativa muy eficiente para resistir los efectos sísmicos en algunos edificios construidos en la zona del lago de Ciudad de México (sobre todo para aquellos con periodo muy cercano al periodo predominante del terreno). Una de las bases para el uso racional de estos sistemas es la definición de factores de reducción que permitan estimar sus fuerzas sísmicas de diseño a partir de un espectro elástico de resistencia de diseño.

Un aspecto relevante durante el diseño sísmico de un sistema estructural integrado por este tipo de dispositivos, es una caracterización adecuada de su capacidad de disipación de energía. En particular, dentro del contexto planteado en este artículo, es posible hacer dicha caracterización a través de un valor de $\xi$. La formalización de este enfoque se da a través del concepto de amortiguamiento viscoso equivalente $\left(\xi_{E Q}\right)$, discutido en detalle por Chopra (2001). Considere la energía disipada en un ciclo de vibración de un dispositivo disipador de energía (información usualmente obtenida experimentalmente), y aquella disipada por un sistema viscoso equivalente durante un ciclo de vibración igual. El valor de $\xi_{E Q}$ se define como aquel valor de $\xi$ que debe asignarse al sistema viscoso equivalente para que ambas energías sean iguales. El concepto de amortiguamiento viscoso equivalente puede extenderse para modelar el amortiguamiento o capacidad de disipación de energía en sistemas de varios grados de libertad, con la particularidad de que es necesario determinar valores de $\xi_{E Q}$ para cada modo de vibración. La extensión del concepto de amortiguamiento equivalente para el diseño sísmico de estructuras con disipadores de energía, ha dado lugar a varios requerimientos formales de diseño sísmico, dentro de los cuales destacan los resumidos en el capítulo 9 de las NEHRP Guidelines for the Seismic Rehabilitation of Buildings (FEMA 273, 1999).

A pesar de que algunos investigadores sugieren que el concepto de amortiguamiento viscoso es aplicable a cualquier tipo de estructura, en algunos casos su aplicación puede llevar a idealizaciones poco confiables. En particular, en muchos casos de interés práctico, la respuesta de una estructura no solo queda definida por su capacidad de disipación de energía, sino por algunas de las particularidades asociadas a su comportamiento histerético. 
Las simplificaciones en las que se incurre cuando se usa el concepto de amortiguamiento viscoso equivalente, aunado a las grandes incertidumbres involucradas en el diseño sísmico (determinación y modelado de las características mecánicas y dinámicas de la estructura, incluyendo $\xi_{E Q}$; la caracterización de la excitación sísmica de diseño y su espectro elástico de resistencia para $\xi$ de 5\%; la estimación de los factores de reducción; etc.), llevan a la necesidad de discutir la aplicabilidad de las expresiones planteadas en este artículo para estimar $R_{\mu \xi}$. En particular, estas expresiones pueden utilizarse durante la concepción y el diseño preliminar de la estructura. Una vez planteado el diseño preliminar, será necesario verificar el desempeño sísmico de la estructura, mediante la simulación numérica detallada de su respuesta dinámica. Esto requiere del análisis estructural de modelos detallados (no lineales de ser necesario) de la estructura, que sean capaces de caracterizar adecuadamente el comportamiento histerético de los disipadores de energía y de los demás elementos estructurales.

Conforme a lo discutido arriba, el diseño sísmico de dispositivos y sistemas disipadores de energía requiere de consideraciones diferentes a las que normalmente se plantean para el diseño de sistemas estructurales tradicionales. En particular, el desempeño sísmico de algunos de estos sistemas innovadores depende de manera importante de parámetros de respuesta tal como las demandas máximas de desplazamiento y velocidad. Dado lo anterior, la incorporación racional de este tipo de dispositivos y sistemas a la práctica común del diseño sísmico en la Ciudad de México solo será posible si se invierten esfuerzos importantes de investigación que conduzcan a cambios sustanciales en las Normas Técnicas Complementarias para Diseño por Sismo.

Las expresiones propuestas en este trabajo no pueden ser incorporadas actualmente a las Normas Técnicas Complementarias para Diseño por Sismo del Reglamento de Construcciones para el D.F. En particular, la forma de los espectros elásticos de resistencia de diseño dentro de estas normas está muy alejada de la forma que exhiben los espectros correspondientes a acelerogramas registrados en diferentes sitios en el D.F.; de tal manera que el uso de las expresiones aquí propuestas en conjunto con los actuales espectros elásticos de diseño llevaría en muchos casos a resultados no conservadores. Para la incorporación racional de expresiones como las aquí propuestas a la normatividad actual, es necesario impulsar un cambio de enfoque en la misma, a partir del cual se promueva la transparencia durante el proceso de diseño sísmico. En este sentido, los autores consideran que los conceptos que han dado lugar al Apéndice A en la última propuesta de actualización para las Normas Técnicas Complementarias para Diseño por Sismo (Ordaz et al. 2000), sientan las bases para un diseño sísmico confiable y transparente, y hacen posible el uso racional de las expresiones aquí propuestas para el diseño sísmico.

\section{CONCLUSIONES}

Aunque a la fecha se han hecho planteamientos importantes para estimar el factor de reducción para diferentes tipos de estructuras, todavía es necesario articular los resultados obtenidos y ampliar su alcance. Al respecto, es necesario enfatizar que no se ha estudiado lo suficiente, particularmente para suelos blandos, la posibilidad de plantear sistemas que con base en una combinación de comportamiento plástico y una capacidad importante de disipación de energía viscosa puedan resistir excitaciones sísmicas intensas. 
El valor del factor de reducción depende de una manera importante del contenido de frecuencias de la excitación sísmica, y del periodo, capacidad máxima de deformación plástica y capacidad de disipación de energía viscosa de la estructura. En este artículo se han propuesto expresiones para estimar, en función de dichos parámetros, el valor del factor de reducción para las zonas de terreno firme y terreno blando del Distrito Federal. Aunque las expresiones propuestas pueden resultar demasiado complejas para algunos ingenieros de la práctica e investigadores, es posible contemplar su simplificación para permitir su uso en la práctica profesional. Dicha simplificación debe plantearse en función de la confiabilidad requerida para el diseño, la incertidumbre involucrada, y lo que se considere en el medio profesional como una complejidad numérica aceptable para un diseño sísmico práctico.

Un aspecto importante en la aplicación de las expresiones propuestas para estimar el factor de reducción, consiste en caracterizar la capacidad de disipación de energía del los dispositivos disipadores de energía mediante un porcentaje de amortiguamiento viscoso crítico. En muchos casos, se obtienen resultados razonables con fines de diseño mediante la aplicación del concepto de amortiguamiento viscoso equivalente, el cual ha sido incorporado en varios requerimientos formales de diseño sísmico (por ejemplo, el FEMA 273).

Las expresiones propuestas para estimar el factor de reducción no pueden aplicarse para estimar las fuerzas sísmicas de diseño a partir del espectro elástico de diseño planteado en el cuerpo de las Normas Técnicas Complementarias para Diseño por Sismo del Reglamento de Construcciones para el D.F. El uso de estas expresiones requiere de una representación más realista de los espectros de resistencia correspondientes a los diferentes sitios y zonas del D.F. Además de lo anterior, se requieren de otros cambios importantes a estas normas, dentro de los cuales destacan una mayor transparencia durante el diseño, y el planteamiento de una revisión detallada del diseño preliminar de la estructura.

\section{AGRADECIMIENTOS}

Los autores deseamos agradecer el apoyo recibido por parte de la Universidad Autónoma Metropolitana, y el patrocinio recibido por parte de la Secretaría General de Obras del Gobierno del Distrito Federal bajo el Convenio 2115/31897.

\section{REFERENCIAS}

Aiken, I D, y J M Kelly (1990), "Earthquake simulator testing and analytical studies of two energy-absorving systems for multistory structures", Reporte No. UCB/EERC-90/03, Universidad de California en Berkeley.

Aiken, I D, D K Nims, A S Whittaker y J M Kelly (1993), “Testing of passive energy dissipation systems", Earthquake Spectra, Vol. 9, No. 3, pp. 335-370.

Arroyo, D (2001), "Factores de reducción de resistencia para sistemas pasivos de disipación de energía", Tesis de Maestría, División de Estudios de Posgrado de la Facultad de Ingeniería, Universidad Autónoma de México, diciembre. 
Badillo, H (2000), "Criterio de diseño sísmico por desempeño para reforzar edificios con disipadores de energía", Tesis de Maestría, División de Estudios de Posgrado de la Facultad de Ingeniería, Universidad Autónoma de México, julio.

Bruneau, M y K Yoshimura (1996), "Damage to masonry buildings caused by the 1995 Hyogoken Nanbu (Kobe, Japan) earthquake”, Canadian Journal of Civil Engineering, Vol. 23, No. 3, pp. 797-807.

Chopra, A K (2001), Dynamics of Structures, Theory and applications to earthquake engineering, segunda edición, Prentice Hall.

FEMA 273 (1999), "NEHRP Guidelines for the seismic rehabilitation of buildings", ASCE/FEMA 273, Federal Emergency Management Agency.

Gaceta Oficial del D.F. (1995), "Normas Técnicas Complementarias para Diseño por Sismo”.

Hanson, R.D (1993), "Supplemental damping for improve the seismic performance", Earthquake Spectra, Vol. 9, No. 3, pp. 319-334.

Hodder, S B (1983), "Computer processing of New Zealand strong-motion accelerograms", Memorias, Third South Pacific Regional Conference on Earthquake Engineering, Vol.1, pp. 3653.

Lai, S P (1982), "Statistical characterization of strong ground motions using power spectral density function", Bulletin of the Seismological Society of America, Vol. 72 , No. 1, pp. 259-274.

Miranda, E (1993), "Site-dependent strength reduction factors", Journal of Structural Engineering, ASCE, Vol. 119, No. 12, pp. 3503-3519.

Mitchell, D (1996), "Damage to concrete structures due to the January 17, 1995, Hyogo-ken Nanbu (Kobe) earthquake", Canadian Journal of Civil Engineering, Vol. 23, No. 3, pp. 757-770

Nassar, A y H Krawinkler (1991), "Seismic demands for SDOF and MDOF systems", Reporte No. 95, The John A. Blume Earthquake Engineering Center, Universidad de Stanford.

Northridge Reconnaissance Team (1996), "Northridge Earthquake Reconnaissance Report", Earthquake Spectra, Supplement C to Volume 11.

Ordaz, M y E Pérez (1998), "Estimation of strength reduction factors for elastoplastic systems: a new approach", Earthquake Engineering and Structural Dynamics, Vol. 27, pp. 889-901.

Ordaz, M, E Miranda y J Avilés (2000), “ Propuesta de Espectros de Diseño por Sismo para el DF", Memorias del XII Congreso Nacional de Ingeniería Estructural, León, CDROM, noviembre

Ordaz, M y C Montoya (2000), “DEGTRA 2000 Ver. 2.0.2”, Instituto de Ingeniería, Universidad Nacional Autónoma de México.

Tajimi, H (1960), "A statistical method for determining the maximum response of a building structure during an earthquake", Memorias, Second World Conference on Earthquake Engineering, Vol. II, pp. 781-797. 
Tena, A y A Vergara (1997), "Comparative study on the seismic retrofit of a mid-rise steel building: steel bracing vs energy dissipation", Earthquake Engineering \& Structural Dynamics, Vol. 26, pp. 637-655.

Trifunac, M D y A G Brady (1975), "A study on the duration of strong earthquake ground motion", Bulletin of the Seimological Society of America, Vol. 65, No. 3, pp. 581-626.

Tung, A, J N Wang, A S Kiremidjian y E Kavazanjian (1992), "Statistical parameters of AM and PSD fuctions for the generation of site-specific strong ground motions", Memorias, Tenth World Conference on Earthquake Engineering, Vol. 2, pp. 867-872. 